\title{
The accuracy and reliability of 3D printed aortic templates: a comprehensive three-dimensional analysis
}

\author{
Pawel Rynio $^{1 \wedge}$, Maciej Wojtuń ${ }^{2}$, Lukasz Wójcik² $^{2}$ Miłosz Kawa ${ }^{2}$,Aleksander Falkowski², Piotr Gutowski ${ }^{1}$, \\ Arkadiusz Kazimierczak ${ }^{1}$ \\ ${ }^{1}$ Department of Vascular Surgery, Pomeranian Medical University in Szczecin, Szczecin, Poland; ${ }^{2}$ Department of Radiology, Pomeranian Medical \\ University in Szczecin, Szczecin, Poland
}

Contributions: (I) Conception and design: P Rynio; (II) Administrative support: P Rynio, A Kazimierczak, P Gutowski, A Falkowski; (III) Provision of study materials or patients: P Rynio, A Kazimierczak; (IV) Collection and assembly of data: P Rynio, A Kazimierczak; (V) Data analysis and interpretation: P Rynio, M Wojtuń, Ł Wójcik; (VI) Manuscript writing: All authors; (VII) Final approval of manuscript: All authors.

Correspondence to: Paweł Rynio, PhD. Department of Vascular Surgery, Pomeranian Medical University in Szczecin, Powstańców Wielkopolskich 72, 70-111, Al. Powstańców Wlkp. 72, Szczecin, Poland. Email: ryniopawel@gmail.com.

Background: Advances in 3D printing technology allow us to continually find new medical applications. One of them is $3 \mathrm{D}$ printing of aortic templates to guide vascular surgeons or interventional radiologists to create fenestrations in the stent-graft surface for the implantation procedure called fenestrated endovascular aortic aneurysm repair. It is believed that the use of 3D printing significantly improves the quality of modified fenestrated stent-grafts. However, the accuracy and reliability of personalized 3D printed models of aortic templates are not well established.

Methods: Thirteen 3D printed templates of the visceral aorta and sixteen of the aortic arch and their corresponding computer tomography of angiography images were included in this accuracy study. The $3 \mathrm{D}$ models were scanned in the same conditions on computed tomography (CT) and evaluated by three physicians experienced in vascular CT assessment. Model and patient CT measurements were performed at key landmarks to maintain quality for stent-graft modification, including side branches and aortic diameters. CT-scanned aortic templates were segmented, aligned with sourced patient data, and evaluated for the Hausdorff matrix. Next, Bland-Altman plots determined the degree of agreement.

Results: The Intraclass Correlation Coefficients values were more than 0.9 for all measurements of aortic diameters and aortic branches diameter in all landmark locations. Therefore, the reliability of the aortic templates was considered excellent. The Bland-Altman plots analysis indicated measurement biases of 0.05 to 0.47 for aortic arch templates and 0.06 to 0.38 for reno-visceral aortic templates. The arithmetic mean of Hausdorff's mean distances of the aortic arch templates was $0.47 \mathrm{~mm}(\mathrm{SD}=0.06)$ and ranged from 0.34 to 0.58 . The mean metrics for abdominal models was $0.24 \mathrm{~mm}(\mathrm{SD}=0.03)$ and ranged from 0.21 to 0.31 .

Conclusions: The printed models of 3D aortic templates are accurate and reliable, thus can be widely used in endovascular surgery and interventional radiology departments as aortic templates to guide the physicianmodified fenestrated stent-graft fabrication.

Keywords: Accuracy; reliability; aneurysm model; aortic template; 3D printing

Submitted May 16, 2021. Accepted for publication Oct 13, 2021.

doi: 10.21037/qims-21-529

View this article at: https://dx.doi.org/10.21037/qims-21-529

\footnotetext{
^ ORCID: 0000-0003-3365-1822.
} 


\section{Introduction}

Over the last two decades, three-dimensional (3D) printing has become a new diagnostic tool in imaging radiology and medicine (1-3). Three-dimensionally printed models of anatomical and pathological structures contain spatial information that would be lost on flat two-dimensional screen monitors. The physical dimension of the $3 \mathrm{D}$ models allows for user interaction and haptic response. These remarkable features quickly became a source of additional information for surgeons. To this day, 3D printing is used to create anatomical models for surgery planning $(2,4,5)$, intraoperative navigation $(2,4)$, procedure simulation (6-8), and resident training $(9,10)$ as well as for producing anatomically realistic, patient-mimicking phantoms for low-contrast lesions detectability testing in radiology departments (11). Its use is estimated to save money and reduce the number of complications (12) in conjunction with augmenting the successful rate of radiological diagnostics of different lesions in patients (11).

The next stage of development and search for new applications for additive manufacturing technologies are $3 \mathrm{D}$ models used as surgical templates or guides (13-15). They are made based on computed tomography (CT) of a given patient, so they are patient-specific devices. Thus, this concept follows a personalized medicine trend-tailored individually to the patient.

One such example is the creation of aortic templates based on computer tomography of angiography (CTA) (16). An aortic template is a hollowed aortic 3D model containing cut off side branches, representing an artificial wall with an empty interior corresponding to the blood pool. Its purpose is to enable the precise design of a constellation of side aortic branches on the stent-graft surface. The template allows performing fenestration on the stent-graft surface so that after its implantation, the fenestration is aligned with the vascular branch. Several smaller series and case descriptions of visceral and aortic arch cases are currently available for patients with threatening aortic aneurysms requiring urgent fenestrated stent-graft implantation (16-19).

Fenestrated and branched stent-grafts are used to extend the indications for endovascular treatment in patients with aortic aneurysms located near the aortic side branches i.e., aneurysms lacking the aortic neck where the regular endograft could anchor. The time required to fabricate a custom-made device is several weeks, and the associated delay makes patients with giant and symptomatic aneurysms unsuitable for this treatment method with custom-made stent-grafts. Therefore, urgent or emergency treatment of patients with abdominal aortic aneurysms is in need of alternative treatment solutions. Another limitation is the relatively high price of custom-made stent-grafts (20). An alternative solution to the above inconveniences is a stentgraft modified by a physician during the operation. Another group of patients convenient for physician-modified stentgrafts (PMSGs) for aortic surgery are those with anatomy unsuitable for commercially available stent-grafts (19) or those with chronic postdissection thoracoabdominal aortic aneurysms (21) or those with small access vessel diameters and access vessel calcifications (22). In addition, the data concerning late advances in PMSGs technologies and medical applications have been recently reviewed elsewhere $(23,24)$. The process of "manual" fenestration design is based on measurements of the aorta and its side branches such as distances, diameters, angles, and angular distances and then transferring them to the stent-graft surfaces in the sterile conditions of the operating suite, thus with limited use of arithmetic tools (25). Radiology workstations and semi-automated analysis tools such as vessel centerline delineation can be helpful. However, the lack of standardization of this method may be one of the factors contributing to significant inter-observer variability during the design process (26).

It is believed that using an artificial aortic template to modify the real stent-graft significantly improves its final quality and reliability (17-19). In recent times, the fenestrated physician-modified stent-grafts started to be an alternative to achieve total endovascular aortic repair, especially in elderly and high-risk patients with major comorbidities. The number of such patients requiring emergency repair is steadily growing because of an aging society. Therefore, the $3 \mathrm{D}$ printing method arouses great hope among vascular surgeons and interventional radiologists to improve their clinical practice results. Therefore, it is expected that with the spread of $3 \mathrm{D}$ printing in the medical sector, this method will replace the "manual" fenestration design of stent-grafts nowadays used increasingly in vascular surgery.

The accuracy of the aortic templates and their ability to reflect the patient's anatomy is crucial for spatially accurate modification of the stent-graft and subsequent precise implantation. If the stent-graft fenestration is performed in the wrong place, it may lead to misalignment with the aortic side branch. As a result, it may lead to aortic branch coverage impairing blood flow or even complete obstruction, causing organ ischemia. 
At present, there is a lack of research that would determine the accuracy of $3 \mathrm{D}$ printed aortic templates. Only a few accuracy studies of aorta and aortic aneurysms have been reported to describe a very small number of cases (27-29). Importantly, it cannot be assumed that the accuracy of 3D models of solid organs and 3D aortic templates will be similar. This is due to an additional stage when preparing the template for the $3 \mathrm{D}$ printing process. An artificial wall is created in a process called modeling. The clear design of the aorta template requires specific additive manufacturing technology. Furthermore, 3D printed aortic templates' accuracy should be established, especially in the key localization for stent-graft modification. Therefore, we aimed here to investigate the accuracy of personalized 3D printed models created for aortic stent-graft modification to support with additional data this novel endovascular method of treatment of aortic aneurysms. We present the following article in accordance with the MDAR checklist (available at https://dx.doi.org/10.21037/qims-21-529).

\section{Methods}

For this study, two groups of patients with aortic pathologies were identified. The first group constituted thirteen patients with juxtarenal abdominal aortic aneurysm treated with fenestrated endovascular aortic aneurysm repair (FEVAR) using physician modified stent-grafts. The operating surgeon performed the modification and included four fenestrations in a personalized 3D printed patient-specific aortic model. The fenestrations aimed to preserve the flow to the celiac artery (CA), the superior mesenteric artery (SMA), the left and right renal artery (LRA, RRA, respectively). Our goal was to anchor a stentgraft proximally to the CA, so we performed fenestrations of all reno-visceral vessels. The second group formed 16 patients with aortic arch and descending aortic aneurysm. The personalized 3D printed models used were prepared with dedication for therapeutic use in FEVAR procedures performed by vascular surgeons. The study was conducted in accordance with the Declaration of Helsinki (as revised in 2013). The study was approved by ethics board of Pomeranian Medical University and individual consent for this retrospective analysis was waived.

\section{Three-dimensional models manufacture}

Three-dimensional personalized printed models were created for all patients enrolled in the study based on preoperative CTAs, which were performed on different CT scanners (Figure 1). CTA scans with a slice thickness exceeding $1 \mathrm{~mm}$ were excluded from the study. The digital $3 \mathrm{D}$ aortic template generation workflow included image processing such as CTA data segmentation, cutting off unnecessary branches, smoothing, and vessel hollowing, thus finally forming the artificial aortic model wall. The first group models presented a limited section of the renovisceral aorta, consisting of a short part of the aorta and cut off ostia of CA, SMA, LRA, and RRA. The second group constituted models of the aortic arch and the proximal part of the descending aorta and cut off ostia of the innominate artery (IA), left common carotid artery (LCCA), and the left subclavian artery (LSA). Two aortic arch cases had a common ostium of IA and LCCA, and were therefore analyzed as single vessel in the study. The external artificial model wall thickness was set to $1.5 \mathrm{~mm}$. The segmentation and modeling were made in 3D Slicer software (version 4.10.0; https://www.slicer. org/) (30). All these steps were executed by a vascular surgeon experienced in vascular segmentation and $3 \mathrm{D}$ printing. Additive manufacturing was performed on a Form 2 printer (Formlabs) using standard clear resin. All models were gas sterilized before use in operating suite, so the analysis performed in this study includes possible geometric distortion due to ethylene oxide. This effect is unspecified by the manufacturer, therefore the material used is not approved for medical sterilization.

\section{Imaging of $3 D$ printed models}

All 3D printed models were scanned using the SOMATOM Definition AS scanner (Siemens). The acquisition was made using vascular presets (BodyAngio) with a slice thickness of $0.75 \mathrm{~mm}$. The patient's names on $3 \mathrm{D}$ models were anonymized to blind evaluating radiologists. For this reason, the acquired scans were named with encrypted code.

\section{Landmark measurements}

The patient CTA (PCTA) scans and model computed tomography (MCT) scans were assessed independently by three physicians well trained in CTA: two radiologists and one vascular surgeon (respectively five, six, and six years of experience in vascular CTA evaluation). The radiologists worked on Syngo.via software (Siemens) using multiplanar reconstruction. To diminish measurement error, we conducted all aortic measurements perpendicular to the 

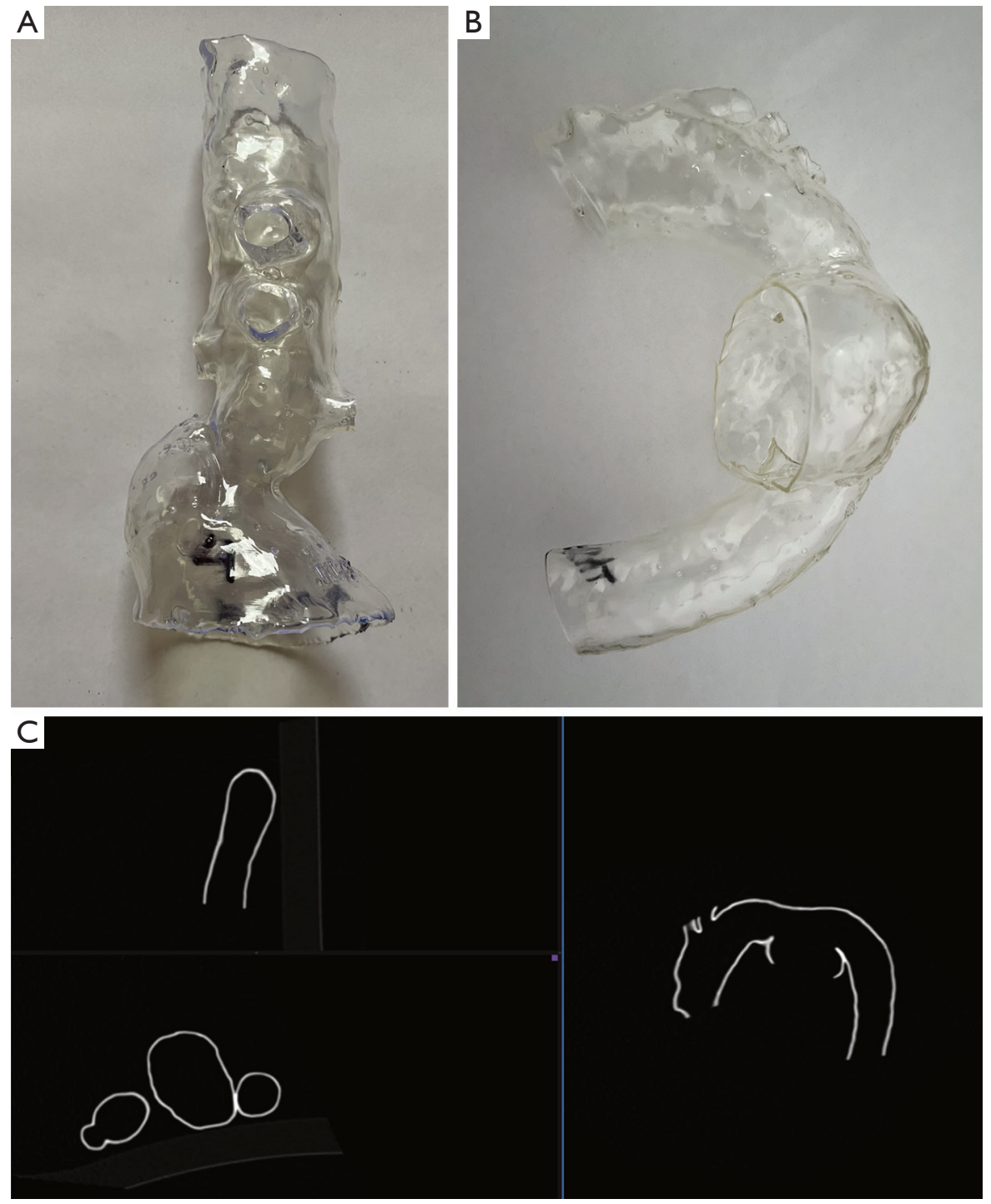

Figure 1 Representative display of three-dimensionally printed aortic templates. A visceral template (A). An aortic arch template (B). Multiplanar reconstruction of CT-scanned aortic arch template (C).

center lumen line. We decided to work on an effective diameter calculated as the arithmetic mean of maximal and minimal vessel diameter. All values were averaged from 3 independent measurements.

The following measurements were conducted on both MCT and PCTA of the aortic arch:

* Aortic diameter 5-cm distally from LSA;

* Aortic diameter 1-cm distally from LSA;

* Aortic diameter at the level of LSA;

* Aortic diameter at the level of LCCA;

* Aortic diameter at the level of IA;

* Diameter of LSA;
* Diameter of LCCA;

* Diameter of IA.

The following measurements were conducted on both MCT and PCTA of the reno-visceral aorta:

* Aortic diameter just below the lowest of the renal arteries;

* Aortic diameter at the level of SMA;

* Aortic diameter at the level of CA;

* Diameter of LRA;

* Diameter of RRA;

* Diameter of SMA;

* Diameter of CA. 
Table 1 Reliability of aortic arch templates

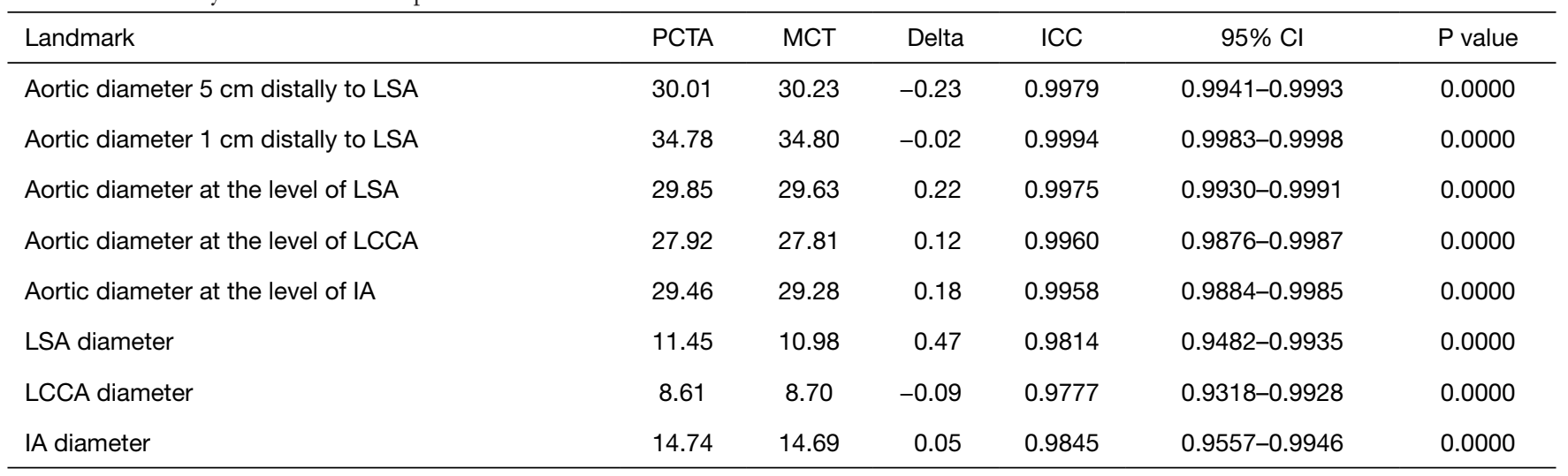

Delta represents the difference between PCT and MCT. PCTA, patient computed tomography of angiography; MCT, model computed tomography; ICC, interclass correlation coefficient; $95 \% \mathrm{CI}$, confidence intervals for ICC; CA, celiac artery; IA, innominate artery; ICC, interclass intercorrelation coefficient; LCCA, left common carotid artery.

\section{Comparisons of meshes}

Additionally, we performed a quality analysis of meshes. To do that, we evaluated stereolithography meshes used to manufacture aortic 3D models with the meshes obtained from CT-scanning of 3D models. To develop the latter, we had to conduct MCT segmentations in 3D Slicer software. Both meshes were aligned through point registration, and Hausdorff distances were assessed in Meshlab free software (31).

\section{Statistical analysis}

To assess the reliability between 3D models and PCTA, we performed the Interclass Intercorrelation Coefficient (ICC) analysis. We used a two-way model for k-raters with an absolute agreement (32). According to the Shapiro-Wilk test, non-normally distributed variables were transformed with Box-Cox transformation and then analyzed by the ICC. Values of less than 0.5 indicate low reliability, values between 0.5 and 0.8 indicate moderate reliability, values between 0.75 and 0.9 good reliability, and values greater than 0.9 indicate excellent reliability. Furthermore, we examined the degree of agreement with Bland-Altman plots with $95 \%$ confidence intervals and 1.96 standard deviation limits. $\mathrm{P}<0.05$ was considered significant. All presented means were calculated as the average of 3 raters. The value of Hausdorff's mean distances were tested for normality and presented as a mean, standard deviation (SD), and value range. All statistical analysis was conducted in PQStat software (PQStat Software) except Box-Cox transformation performed in Statistica 13.0 (TIBCO Software).

\section{Results}

\section{Three-dimensional models reliability}

The ICC values were greater than 0.9 for all measurements of aortic diameters and aortic branch diameter in all landmark locations (Tables 1,2). The ICC analysis was statistically significant for all values. Therefore, the reliability of the aortic templates was considered excellent. However, when extrapolating the data for the larger population, one should mind the confidence intervals. The lower limit was more significant than 0.9 in all landmarks except visceral and renal branches. The absolute difference between PCTA and MCT for landmark measurements ranged from 0.05 to 0.47 for aortic arch templates and from 0.06 to 0.38 for reno-visceral aortic templates.

\section{Degree of agreement}

The Bland-Altman plots analysis indicated measurement biases of 0.06 to 0.38 for reno-visceral aortic templates and 0.05 to 0.47 for aortic arch templates (Figures 2,3). However, the Bland and Altman method does not state if such bias is relevant or not. It should be judged by the clinicians and interpreted to the specific clinical situation. Considering the spatial resolution of CT scanners greater than $0.75 \mathrm{~mm}$, one should conclude that features smaller than that cannot be reliably visualized. Therefore, we could consider $0.75 \mathrm{~mm}$ as the upper limit for the bias. The analysis of the graphs 
Table 2 Reliability of visceral aortic templates

\begin{tabular}{|c|c|c|c|c|c|c|}
\hline Landmark & PCTA & MCT & Delta & ICC & $95 \% \mathrm{Cl}$ & $P$ value \\
\hline Aortic diameter below SMA & 26.82 & 26.50 & 0.32 & 0.9961 & $0.9867-0.9988$ & 0.0000 \\
\hline Aortic diameter above CA & 26.75 & 26.37 & 0.38 & 0.9888 & $0.9463-0.9969$ & 0.0000 \\
\hline CA diameter & 6.58 & 6.22 & 0.36 & 0.9401 & $0.6009-0.9849$ & 0.0000 \\
\hline LRA diameter & 6.21 & 5.89 & 0.32 & 0.9630 & $0.8219-0.9898$ & 0.0000 \\
\hline RRA diameter & 5.86 & 5.78 & 0.08 & 0.9686 & $0.8979-0.9904$ & 0.0000 \\
\hline
\end{tabular}

Delta represents the difference between PCT and MCT. PCTA, patient computed tomography of angiography; MCT, model computed tomography; ICC, interclass correlation coefficient; $95 \% \mathrm{Cl}$, confidence intervals for ICC; LRA, left renal artery; RRA, right renal artery; CA, celiac artery; SMA, superior mesenteric artery.

does not indicate any trend or systemic error for the difference of measurements. The variability is consistent across the plot.

\section{Hausdorff distances}

The arithmetic mean of Hausdorff's mean distances of the aortic arch templates was $0.47 \mathrm{~mm}$ (SD 0.06) and ranged from 0.34 to 0.58 . The mean metrics for abdominal models was $0.24 \mathrm{~mm}$ (SD 0.03 ) and ranged from 0.21 to 0.31 . The exemplary models with overlayed heatmaps of Hausdorff metrics are presented in Figures 4,5.

\section{Discussion}

Here, we evaluated the 3D aortic template accuracy based on landmark measurements. We selected measurement points located in the key locations for stent-graft modification. Any deviations from the pattern in these locations may negatively affect the operation course. However, the verification of the accuracy of the model itself is also burdened with measurement error. The analysis of the model's accuracy consists of the real error plus the measurement error (33). It could be assumed that the measurement error on both the model and the tomography imaging will be abolished with a sufficient number of measurements. This hypothesis assumes that the error size is the same on the model as on the CT scans. This does not necessarily have to be true because the scanned $3 \mathrm{D}$ model gives a different radiological shadow. Additionally, the 3D model is static, while the patient's aorta is in motion, generating wall movement and blurring and negatively affecting the accuracy of measurements.

Previously, intraobserver differences and variability on CTA measurements of aortic diameter have been established in a few studies $(34,35)$. The mean arithmetic difference for one reader was $-0.21 \mathrm{~mm}$, while for the other was $0.2 \mathrm{~mm}$. These values are similar to those obtained in our study. Intraobserver variability for aortic measurements can be as high as 2.6 to $3.8 \mathrm{~mm}$ (34). For this reason, the accuracy of the model cannot be assessed based on individual measurements. It always must be a series that averages the error of measurement. The threshold from which a model is considered inaccurate should be the voxel's size of the tomography used to scan a $3 \mathrm{D}$ model. The results obtained in this study may be used as a reference for the implementation of $3 \mathrm{D}$ model quality assurance protocols in units dealing with this subject.

The comparison of our results to other research reports is difficult due to the different methodology applied. The $3 \mathrm{D}$ models mainly differ in the additive manufacturing technology, materials used for fabrication, the type and quality of CT input data, type of pathology, 3D model destination, and finally, the method of measurement. D. Ho et al. fabricated two models of the dissected thoracic aorta (29). The models were made in the Fused Deposition Modelling (FDM) technology; thus, they were opaque. One physician evaluated specified landmarks based on horizontal and vertical diameter. The mean difference was equal to $1.0 \mathrm{~mm}$ (SD 1.0) and $1.2 \mathrm{~mm}$ (SD 0.9) for the first and second model. The other group assessed the accuracy of $3 \mathrm{D}$ aortic models manufactured for simulation purposes (28). The models were equipped with outlets and inlets for the connection to the perfusion system. Two raters assessed six 

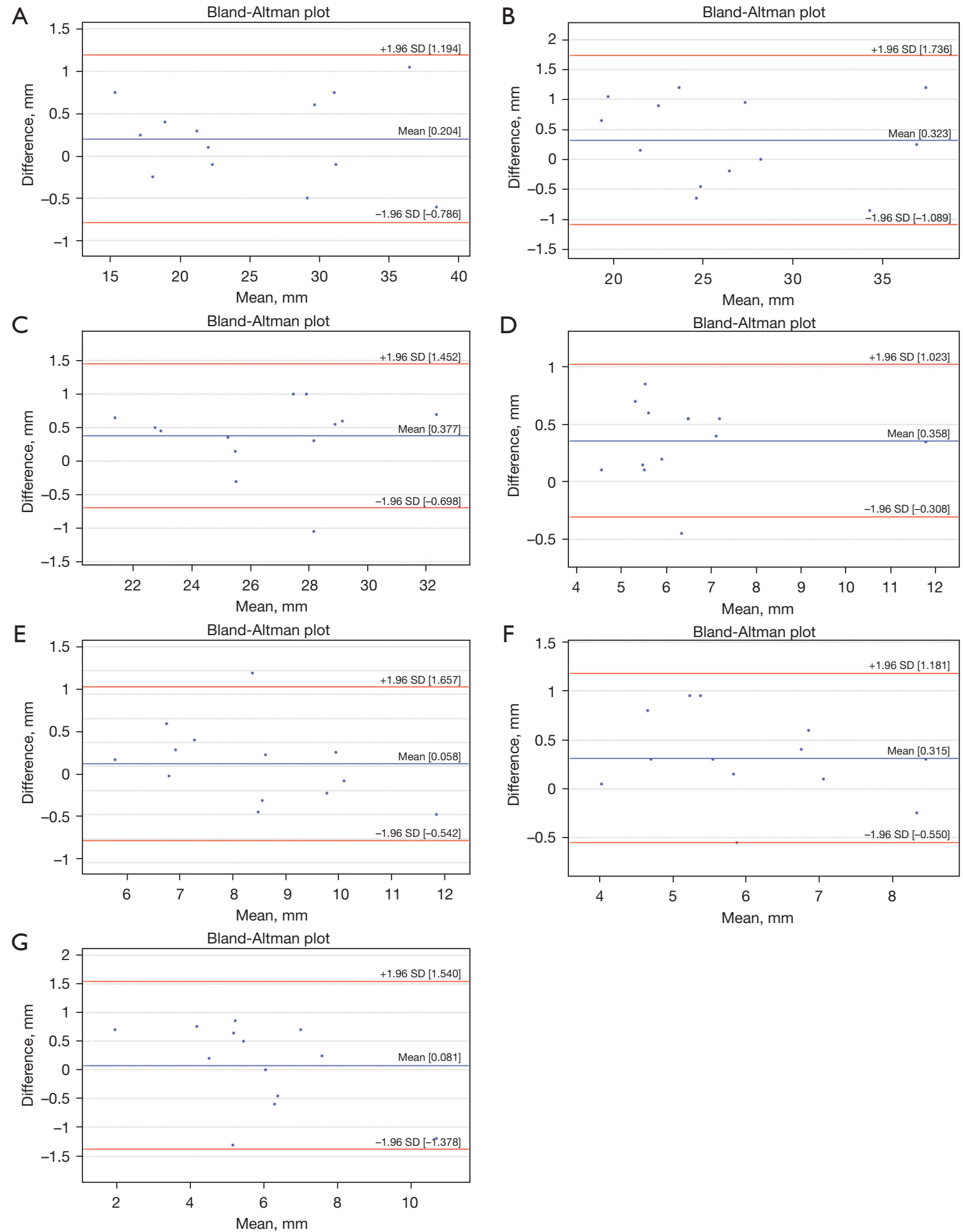

Figure 2 Bland-Altman plots showing the degree of agreement between CTA and 3D model for abdominal aortic diameter measured at the level of key landmarks and the degree of agreement of abdominal aortic branch diameters. (A) The degree of agreement at the level of the lowest renal artery; (B) the degree of agreement at the level of the superior mesenteric artery; (C) the degree of agreement above the celiac trunk; (D) the degree of agreement of the celiac artery diameter; (E) the degree of agreement of the superior mesenteric artery diameter; (F) the degree of agreement of the left renal artery diameter; $(\mathrm{G})$ the degree of agreement of the right renal artery diameter. CTA, computer tomography of angiography. 
A

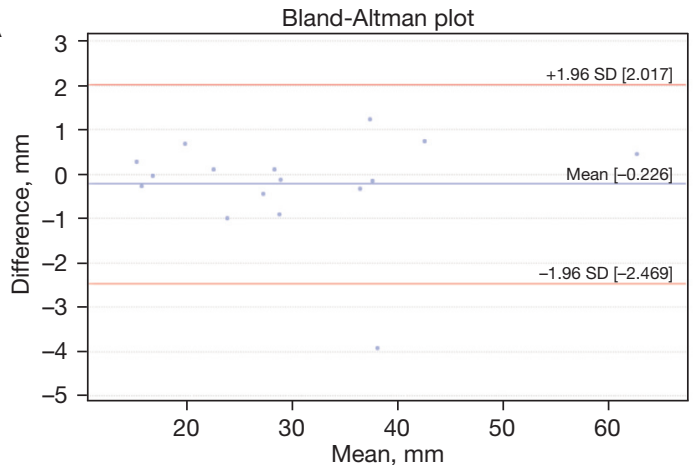

C

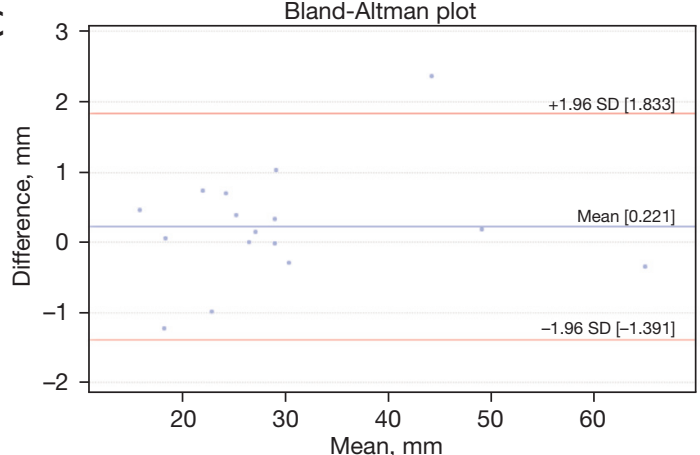

E

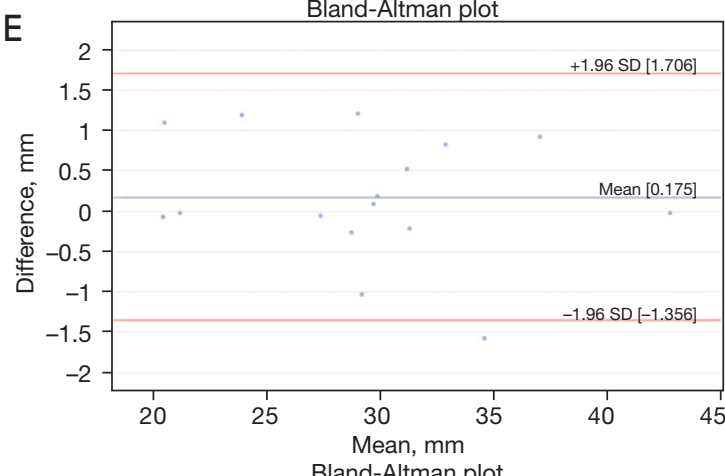

G

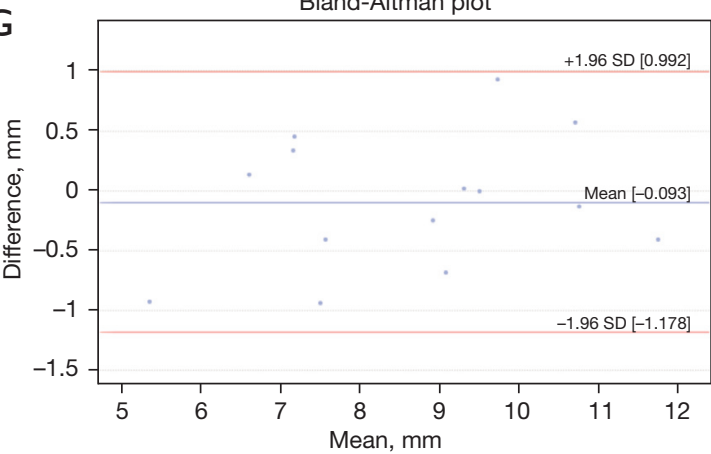

B
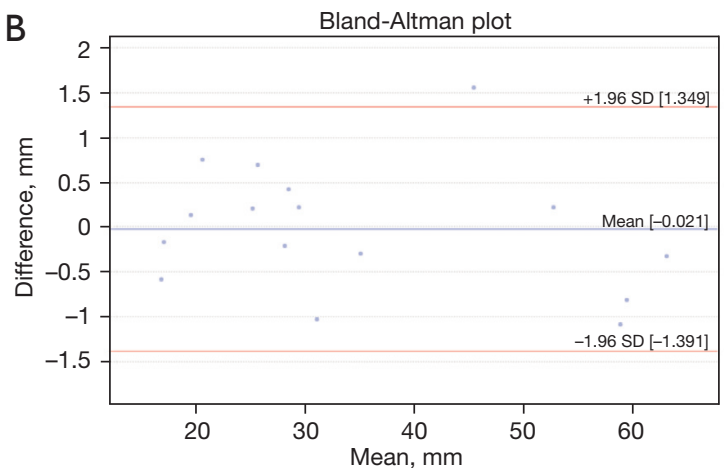

D
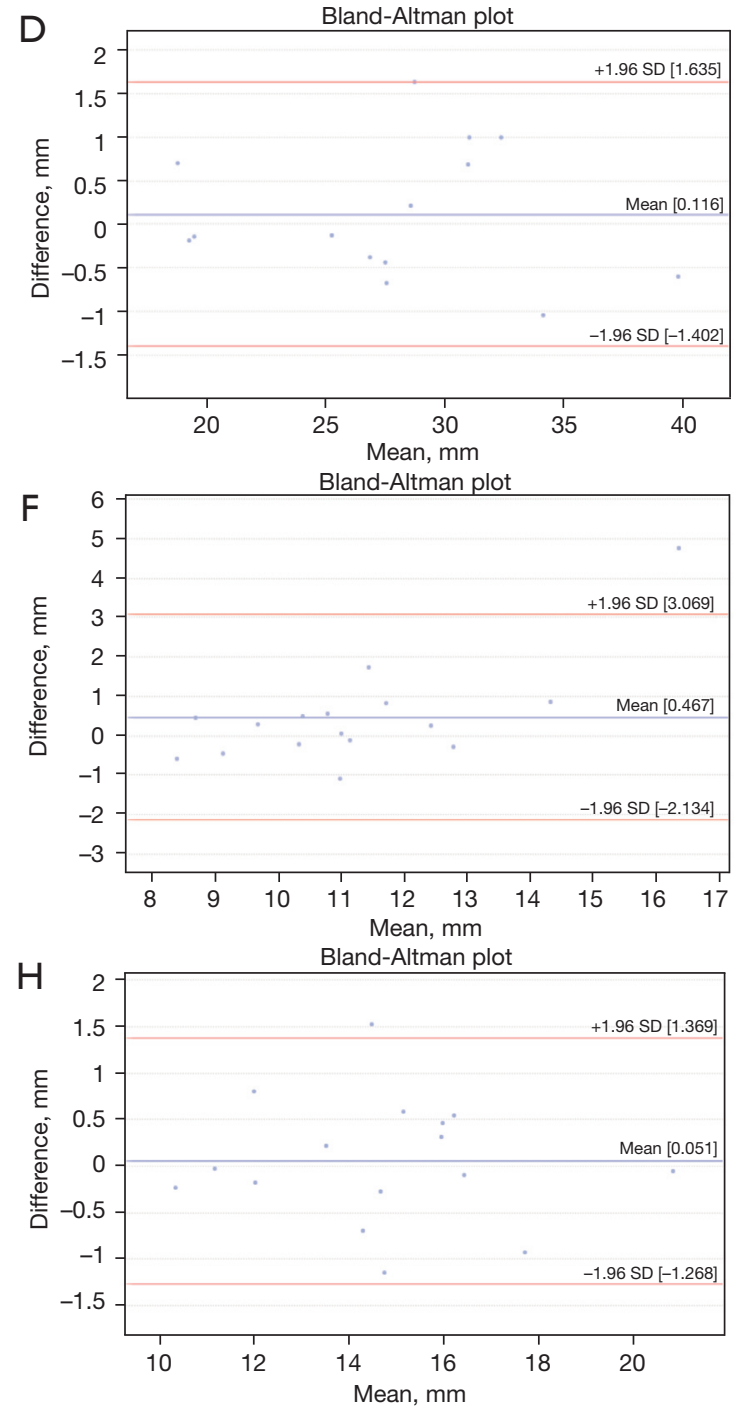

Figure 3 Bland-Altman plots showing the degree of agreement between CTA and 3D model for thoracic aortic diameter at the level of key landmarks and the degree of agreement of aortic arch branch diameters. (A) The degree of agreement measured 5-cm distally from LSA; (B) the degree of agreement 1-cm distally from LSA; (C) the degree of agreement at the level of LSA; (D) the degree of agreement at the level of LCCA; (E) the degree of agreement at the level of IA; (F) the degree of agreement of LSA diameter; (G) the degree of agreement of LCCA diameter; (H) the degree of agreement of IA diameter. CTA, computer tomography of angiography; LSA, left subclavian artery; LCCA, left common carotid artery; IA, innominate artery. 

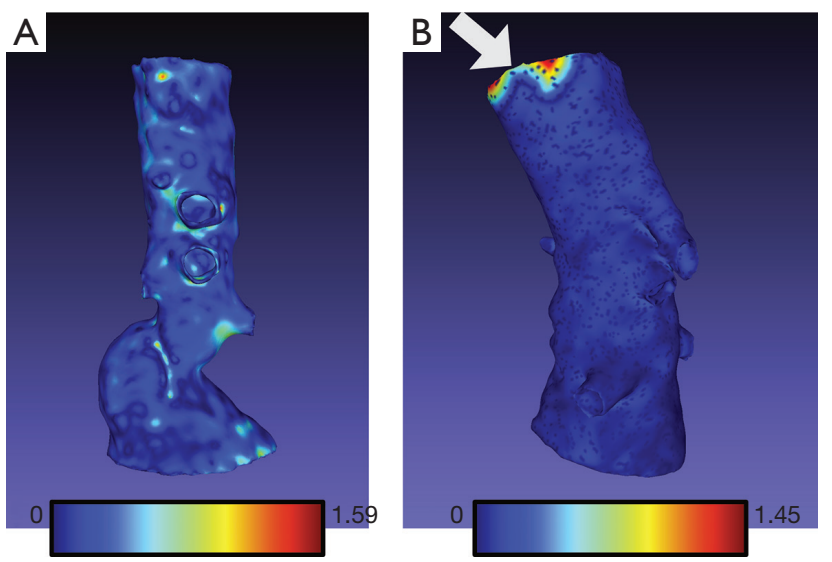

Figure 4 Heatmaps showing Hausdorff's distances for exemplary two reno-visceral aortic model templates. The minimal and maximal values are color-coded and presented in $\mathrm{mm}$. The white arrow points out the model edge, which broke off during use. (A) First exemplary model; (B) second exemplary model.

abdominal aortoiliac aneurysm models fabricated in PolyJet technology. The mean deviations of 3D replicas ranged from -0.73 to $0.14 \mathrm{~mm}$. Besides, Kato et al. fabricated five abdominal aortic aneurysm models in SLA technology. The mean difference was $-0.2(0.4 \mathrm{SD})$ with a significant correlation between 3D models and patient CT data (27). It is important to note that the type of $3 \mathrm{D}$ printing technology itself affects the quality of cardiovascular models among which vat polymerization and material jetting appear to be the most accurate when compared using heatmap methods (36).

Fabrication of medical 3D models is a complex multistage process. The first step is data imaging in DICOM viewers and organ segmentation. After converting the data to the Standard Tessellation Language (STL) format, next, it is possible to model the data to clinically required $3 \mathrm{D}$ forms and functions. Before sending the blueprint to a $3 \mathrm{D}$ printer, the last step is to design model supports and plan the $3 \mathrm{D}$ printer's building chamber's spatial arrangement. The fabricated model requires postprocessing, which usually means removing the unnecessary supports and smoothing the model surface. In each of these steps, a quality error can occur. Moreover, the error that occurred in one step can be additionally augmented in other of the subsequent stages. For this reason, it is crucial to establish maximum permissible deviations and quality standards for specific types of models. This data must be determined before the introduction of quality assurance programs to
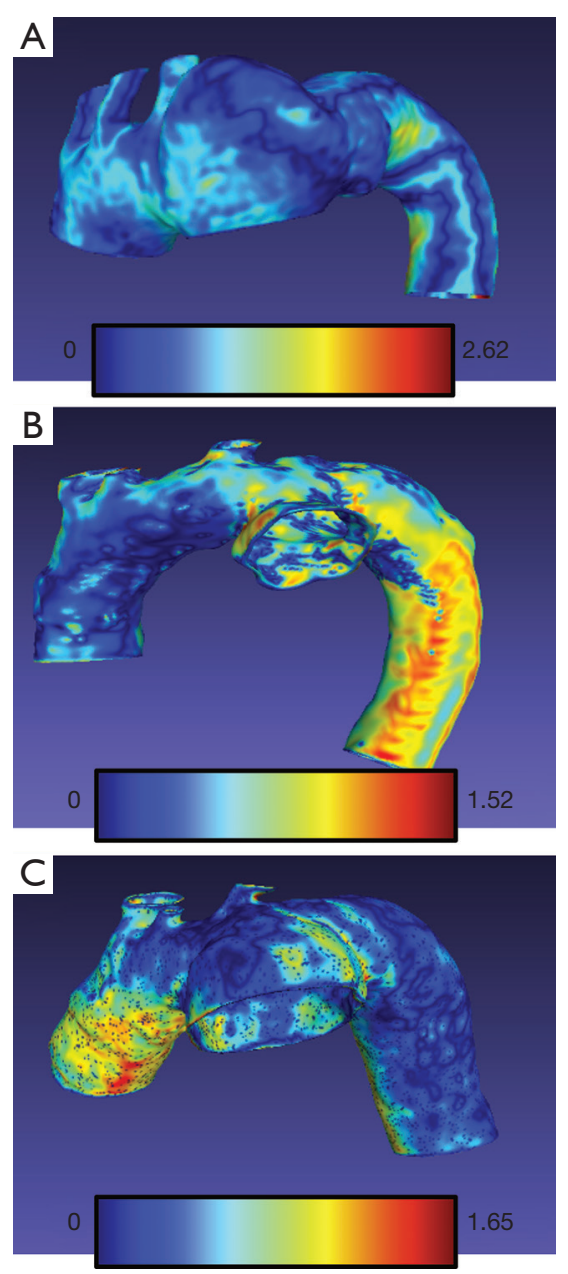

Figure 5 Heatmaps showing Hausdorff distances for three exemplary aortic arch templates. The minimal and maximal values are color-coded and presented in mm. (A) First exemplary model; (B) second exemplary model; (C) third exemplary model.

the medical practice.

$3 \mathrm{D}$ aortic templates should be fabricated in a technology that ensures at least partial wall transparency so that the surgeon can adequately assess the stent-graft structure and its wall apposition (37). This prerequisite limits the range of technologies used in additive manufacturing, disqualifying popular FDM (38). PolyJet and SLA stereolithography used in this study are the technologies of choice. Therefore, inaccuracies in $3 \mathrm{D}$ printing will be due to the limitations of a specific 3D printer (39). By narrowing the number of available technologies, we can identify defects and potential points of error in the selected procedure. Inaccuracy of 3D printing may also result from the limitations of a given technology (40). For example, the laser spot size on Form 2 
is 140 microns, which means that it cannot generate smaller size parts in the $\mathrm{XY}$ plane. The layer thickness in the $\mathrm{Z}$-axis is adjustable and ranges from 25 to 100 microns. Thus, both parameters determine the final three-dimensional resolution of the printer.

Vascular centers performing stent-graft modifications using $3 \mathrm{D}$ printed templates should implement appropriate quality control standards. Developed procedures should consider the type of additive manufacturing technology used, segmentation and digital modeling methods, postprocessing of the model, and the impact of sterilization. The model's accuracy should be checked at the stage of the digital blueprint and the physical 3D model. A good practice would be to engage an independent evaluator who was not involved in the model development process. The results of this study can serve as a reference when evaluating the accuracy of individual 3D templates.

\section{Conclusions}

Personalized 3D printed aortic templates are accurate and reliable models, thus can be used in endovascular surgery as aortic templates to guide fenestrated physician-modified branched stent-graft fabrication. We postulate that the vascular centers performing stent-grafts modifications using $3 \mathrm{D}$ printing should establish their own and independent systems for management and quality control of the $3 \mathrm{D}$ models performed for using them routinely in clinical practice in operating theaters.

\section{Acknowledgments}

Funding: None.

\section{Footnote}

Reporting Checklist: The authors have completed the MDAR checklist. Available at https://dx.doi.org/10.21037/qims-21529

Conflicts of Interest: All authors have completed the ICMJE uniform disclosure form (available at https://dx.doi. org/10.21037/qims-21-529). The authors have no conflicts of interest to declare.

Ethical Statement: The authors are accountable for all aspects of the work in ensuring that questions related to the accuracy or integrity of any part of the work are appropriately investigated and resolved. The study was conducted with complete anonymity for retrospectively analyzed patient data. The study was conducted in accordance with the Declaration of Helsinki (as revised in 2013). The study was approved by ethics board of Pomeranian Medical University and individual consent for this retrospective analysis was waived.

Open Access Statement: This is an Open Access article distributed in accordance with the Creative Commons Attribution-NonCommercial-NoDerivs 4.0 International License (CC BY-NC-ND 4.0), which permits the noncommercial replication and distribution of the article with the strict proviso that no changes or edits are made and the original work is properly cited (including links to both the formal publication through the relevant DOI and the license). See: https://creativecommons.org/licenses/by-nc-nd/4.0/.

\section{References}

1. Pugliese L, Marconi S, Negrello E, Mauri V, Peri A, Gallo V, Auricchio F, Pietrabissa A. The clinical use of 3D printing in surgery. Updates Surg 2018;70:381-8.

2. Tack P, Victor J, Gemmel P, Annemans L. 3D-printing techniques in a medical setting: a systematic literature review. Biomed Eng Online 2016;15:115.

3. Hachulla AL, Noble S, Guglielmi G, Agulleiro D, Müller H, Vallée JP. 3D-printed heart model to guide LAA closure: useful in clinical practice? Eur Radiol 2019;29:251-8.

4. Pietrabissa A, Marconi S, Negrello E, Mauri V, Peri A, Pugliese L, Marone EM, Auricchio F. An overview on 3D printing for abdominal surgery. Surg Endosc 2020;34:1-13.

5. Witowski J, Budzyński A, Grochowska A, Ballard DH, Major P, Rubinkiewicz M, Złahoda-Huzior A, Popiela TJ, Wierdak M, Pędziwiatr M. Decision-making based on $3 \mathrm{D}$ printed models in laparoscopic liver resections with intraoperative ultrasound: a prospective observational study. Eur Radiol 2020;30:1306-12.

6. Mafeld S, Nesbitt C, McCaslin J, Bagnall A, Davey P, Bose P, Williams R. Three-dimensional (3D) printed endovascular simulation models: a feasibility study. Ann Transl Med 2017;5:42.

7. Rynio P, Falkowski A, Witowski J, Kazimierczak A, Wójcik Ł, Gutowski P. Simulation and Training of Needle Puncture Procedure with a Patient-Specific 3D Printed Gluteal Artery Model. J Clin Med 2020;9:686.

8. Jahnke P, Schwarz FB, Ziegert M, Almasi T, Abdelhadi 
O, Nunninger M, Hamm B, Scheel M. A radiopaque 3D printed, anthropomorphic phantom for simulation of CTguided procedures. Eur Radiol 2018;28:4818-23.

9. Torres I, De Luccia N. Artificial vascular models for endovascular training (3D printing). Innov Surg Sci 2018;3:225-34.

10. Torres IO, De Luccia N. A simulator for training in endovascular aneurysm repair: The use of three dimensional printers. Eur J Vasc Endovasc Surg 2017;54:247-53.

11. Ardila Pardo GL, Conzelmann J, Genske U, Hamm B, Scheel M, Jahnke P. 3D printing of anatomically realistic phantoms with detection tasks to assess the diagnostic performance of CT images. Eur Radiol 2020;30:4557-63.

12. Fan G, Meng Y, Zhu S, Ye M, Li M, Li F, Ye Y, Liu $\mathrm{Z}$, Weiqin H, Xie Y. Three-dimensional printing for laparoscopic partial nephrectomy in patients with renal tumors. J Int Med Res 2019;47:4324-32.

13. Marinescu R, Popescu D, Laptoiu D. A Review on 3D-Printed Templates for Precontouring Fixation Plates in Orthopedic Surgery. J Clin Med 2020;9:2908.

14. Oth O, Durieux V, Orellana MF, Glineur R. Genioplasty with surgical guide using $3 \mathrm{D}$-printing technology: A systematic review. J Clin Exp Dent 2020;12:e85-92.

15. Pepper J, Golesworthy T, Utley M, Chan J, Ganeshalingam S, Lamperth M, Mohiaddin R, Treasure T. Manufacturing and placing a bespoke support for the Marfan aortic root: description of the method and technical results and status at one year for the first ten patients. Interact Cardiovasc Thorac Surg 2010;10:360-5.

16. Rynio P, Kazimierczak A, Jedrzejczak T, Gutowski P. A 3-Dimensional Printed Aortic Arch Template to Facilitate the Creation of Physician-Modified Stent-Grafts. J Endovasc Ther 2018;25:554-8.

17. Tong YH, Yu T, Zhou MJ, Liu C, Zhou M, Jiang Q, Liu CJ, Li XQ, Liu Z. Use of 3D Printing to Guide Creation of Fenestrations in Physician-Modified Stent-Grafts for Treatment of Thoracoabdominal Aortic Disease. J Endovasc Ther 2020;27:385-93.

18. Tong Y, Qin Y, Yu T, Zhou M, Liu C, Liu C, Li X, Liu Z. Three-Dimensional Printing to Guide the Application of Modified Prefenestrated Stent Grafts to Treat Aortic Arch Disease. Ann Vasc Surg 2020;66:152-9.

19. Branzan D, Geisler A, Grunert R, Steiner S, Bausback Y, Gockel I, Scheinert D, Schmidt A. The Influence of 3D Printed Aortic Models on the Evolution of Physician Modified Stent Grafts for the Urgent Treatment of Thoraco-abdominal and Pararenal Aortic Pathologies. Eur
J Vasc Endovasc Surg 2021;61:407-12.

20. Hart JP. Conceptual, cost, regulatory, material properties, and sterilization considerations in three-dimensional printed template-assisted physician modified stent grafts for fenestrated endovascular aneurysm repair. J Vasc Surg 2021;74:307-8.

21. Yang G, Zhang M, Zhang Y, Du X, Qiao T, Li X, Zhou M. Endovascular Repair of Postdissection Aortic Aneurysms Using Physician-Modified Endografts. Ann Thorac Surg 2021;112:1201-8.

22. Paludetto G, Van der Meulen S, Ouriel K, Patarca R. Physician Modified Low Profile Endograft for Endovascular Repair of Juxtarenal Abdominal Aortic Aneurysms in Patients with Small Access Vessels. EJVES Vasc Forum 2021;51:9-12.

23. Coles-Black J, Barber T, Bolton D, Chuen J. A systematic review of three-dimensional printed templateassisted physician-modified stent grafts for fenestrated endovascular aneurysm repair. J Vasc Surg 2021;74:296306.e1.

24. Canonge J, Jayet J, Heim F, Chakfé N, Coggia M, Coscas $\mathrm{R}$, Cochennec F. Comprehensive Review of Physician Modified Aortic Stent Grafts: Technical and Clinical Outcomes. Eur J Vasc Endovasc Surg 2021;61:560-9.

25. Oderich GS, Ribeiro M, Reis de Souza L, Hofer J, Wigham J, Cha S. Endovascular repair of thoracoabdominal aortic aneurysms using fenestrated and branched endografts. J Thorac Cardiovasc Surg 2017;153:S32-S41.e7.

26. Koleilat I, Jaeggli M, Ewing JA, Androes M, Simionescu DT, Eidt J. Interobserver variability in physicianmodified endograft planning by comparison with a three-dimensional printed aortic model. J Vasc Surg 2016;64:1789-96.

27. Kato K, Ishiguchi T, Maruyama K, Naganawa S, Ishigaki T. Accuracy of plastic replica of aortic aneurysm using 3D-CT data for transluminal stent-grafting: experimental and clinical evaluation. J Comput Assist Tomogr 2001;25:300-4.

28. Kaschwich M, Horn M, Matthiensen S, Stahlberg E, Behrendt CA, Matysiak F, Bouchagiar J, Dell A, Ellebrecht D, Bayer A, Kleemann M. Accuracy evaluation of patient-specific 3D-printed aortic anatomy. Ann Anat 2021;234:151629.

29. Ho D, Squelch A, Sun Z. Modelling of aortic aneurysm and aortic dissection through 3D printing. J Med Radiat Sci 2017;64:10-7.

30. Fedorov A, Beichel R, Kalpathy-Cramer J, Finet J, Fillion- 
Robin JC, Pujol S, Bauer C, Jennings D, Fennessy F, Sonka M, Buatti J, Aylward S, Miller JV, Pieper S, Kikinis R. 3D Slicer as an image computing platform for the Quantitative Imaging Network. Magn Reson Imaging 2012;30:1323-41.

31. Cignoni P, Callieri M, Corsini M, Dellepiane M, Ganovelli F, Ranzuglia G. MeshLab: an Open-Source Mesh Processing Tool. Eurographics Italian Chapter Conference. MeshLab: an Open-Source Mesh Processing Tool I Semantic Scholar. Available online: https://www. semanticscholar.org/paper/MeshLab\%3A-an-OpenSource-Mesh-Processing-Tool-Cignoni-Callieri/1a3e542 b908e6af7923b04d4738e45e5bac10dcb. Accessed 18 Nov 2020.

32. Koo TK, Li MY. A Guideline of Selecting and Reporting Intraclass Correlation Coefficients for Reliability Research. J Chiropr Med 2016;15:155-63.

33. Etemad-Shahidi Y, Qallandar OB, Evenden J, AlifuiSegbaya F, Ahmed KE. Accuracy of 3-Dimensionally Printed Full-Arch Dental Models: A Systematic Review. J Clin Med 2020;9:3357.

34. Singh K, Jacobsen BK, Solberg S, Bønaa KH, Kumar S, Bajic R, Arnesen E. Intra- and interobserver variability in the measurements of abdominal aortic and common iliac artery diameter with computed tomography. The Tromsø study. Eur J Vasc Endovasc Surg 2003;25:399-407.

Cite this article as: Rynio P, Wojtuń M, Wójcik $Ł$, Kawa M, Falkowski A, Gutowski P, Kazimierczak A. The accuracy and reliability of $3 \mathrm{D}$ printed aortic templates: a comprehensive three-dimensional analysis. Quant Imaging Med Surg 2022;12(2):1385-1396. doi: 10.21037/qims-21-529
35. Aarts NJ, Schurink GW, Schultze Kool LJ, Bode PJ, van Baalen JM, Hermans J, van Bockel JH. Abdominal aortic aneurysm measurements for endovascular repair: intraand interobserver variability of CT measurements. Eur J Vasc Endovasc Surg 1999;18:475-80.

36. Otton JM, Birbara NS, Hussain T, Greil G, Foley TA, Pather N. 3D printing from cardiovascular CT: a practical guide and review. Cardiovasc Diagn Ther 2017;7:507-26.

37. Kirby B, Kenkel JM, Zhang AY, Amirlak B, Suszynski TM. Three-dimensional (3D) synthetic printing for the manufacture of non-biodegradable models, tools and implants used in surgery: a review of current methods. J Med Eng Technol 2021;45:14-21.

38. Guessasma S, Belhabib S, Bassir D, Nouri H, Gomes S. On the Mechanical Behaviour of Biosourced Cellular Polymer Manufactured Using Fused Deposition Modelling. Polymers (Basel) 2020;12:2651.

39. Rungrojwittayakul O, Kan JY, Shiozaki K, Swamidass RS, Goodacre BJ, Goodacre CJ, Lozada JL. Accuracy of 3D Printed Models Created by Two Technologies of Printers with Different Designs of Model Base. J Prosthodont 2020;29:124-8.

40. Zhang ZC, Li PL, Chu FT, Shen G. Influence of the three-dimensional printing technique and printing layer thickness on model accuracy. J Orofac Orthop 2019;80:194-204. 